\title{
Determination and Application of Double Ellipsoid Heat Source Model Parameters
}

\author{
Rui-ying $\mathrm{Li}^{\mathrm{a}}$, Chunyu Chen ${ }^{\mathrm{b}}$, Dawei Zhao ${ }^{\mathrm{c}}$, and Chunmei $\mathrm{Wu}{ }^{\mathrm{d}}$ \\ College of Mechanical \& Electronic Engineering, Daqing Normal University, Daqing 163712, China.

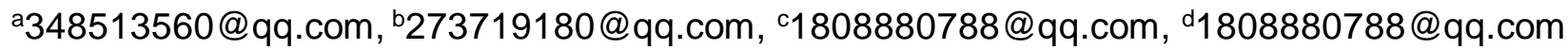

Keywords: double ellipsoid heat mode, gas tungsten arc welding, numerical simulation

\begin{abstract}
Based on the double ellipsoid thermal source model, the numerical analysis model of the 3d transient welding thermal process of the GTAW welding stainless steel $0 \mathrm{Cr} 18 \mathrm{Ni} 9$ thin plate was established. The method for determining the heat source distribution parameters by the calibration tool of SYSWELD software is given. The finite element solution of the molten pool is compared with the experimental results, and the calculated results are in good agreement with the measured results.
\end{abstract}

\section{Preface}

Gas Tungsten Arc Welding is commonly used in the automatic welding and robot welding of one of the welding process. Some researchers have established corresponding models for the two-dimensional transient or $3 \mathrm{~d}$ quasi-steady state problems of fixed arc [1,2]. And most of them are in planar heat source mode, and more and more researchers are paying attention to the $3 \mathrm{~d}$ transient behavior of welding pool and have achieved good results [3].

Is proposed in this paper the double ellipsoid heat source model can well reflect the power density distribution of GTAW welding arc, because there is no clear formula to determine the double ellipsoid body heat source distribution parameter value, how to choose, need a lot of trial process to get reasonable distribution of heat source parameter value. This paper analyzes reasons for this, gives the application of SYSWELD software calibration tool to determine the double ellipsoid body heat source distribution parameter of the method; Using correction moving double ellipsoid heat source model, under the action of the arc movement of GTAW welding $3 \mathrm{~mm}$ thick stainless steel sheet welding process has carried on the finite element simulation, predict the three-dimensional transient when plate welding molten pool in the shape of dynamic evolution, and test verification.

\section{Mathematical Model}

\subsection{Temperature Field Control Equation and Boundary Condition}

Welding material is isotropic, temperature $\mathrm{T}(\mathrm{x}, \mathrm{y}, \mathrm{z}$, and $\mathrm{t})$ is the position coordinates $(\mathrm{x}, \mathrm{y}$, and $\mathrm{z})$ as a function of time $t$, the indirect thinking of molten pool to the liquid metal flow, under the condition of arbitrary point in the region should satisfy the equation of conservation of energy:

$$
\rho c_{p} \frac{\partial T}{\partial t}=\frac{\partial}{\partial x}\left(k \frac{\partial T}{\partial x}\right)+\frac{\partial}{\partial y}\left(k \frac{\partial T}{\partial y}\right)+\frac{\partial}{\partial z}\left(k \frac{\partial T}{\partial z}\right)+Q
$$

In the formula: $\mathrm{k}$ for the thermal conductivity; $\mathrm{c}_{\mathrm{p}}$ is constant pressure specific heat capacity; $\rho$ is the density of the material; $\mathrm{T}$ is temperature; $\mathrm{t}$ is time; $\mathrm{Q}$ is the source term.

The boundary condition of the energy conservation equation:

On the top surface of the work-piece:

$$
k \frac{\partial T}{\partial z}=q_{\mathrm{a}}-q_{\mathrm{cr}}-q_{\mathrm{evp}} \quad q_{\mathrm{cr}}=\alpha_{\mathrm{cr}}\left(T-T_{a}\right) \quad q_{\mathrm{evp}}=m_{\mathrm{er}} L_{\mathrm{b}}
$$

Type: $\mathrm{q}_{\mathrm{a}}$ is the arc heat flux density; $\mathrm{Q}_{\mathrm{cr}}$ is the heat flux density caused by convection and radiation. $Q_{\text {evp }}$ is the heat flux density dissipated by evaporation; the synthesis heat conductivity of $\alpha_{\mathrm{cr}}$ for convection and radiation; $\mathrm{T}_{\mathrm{a}}$ is the ambient temperature; $\mathrm{M}_{\mathrm{er}}$ is the evaporation rate; $\mathrm{L}_{\mathrm{b}}$ is the latent heat 
constant of vaporization. For stainless steel, liquid gas phase change latent heat is $\mathrm{L}_{b}=6.2595 \times 10^{6}$ $\left(\mathrm{J} \cdot \mathrm{kg}^{-1}\right)$.

Under the surface of the work-piece:

$$
-k \frac{\partial T}{\partial z}=-q_{\mathrm{cr}}
$$

The calculation area is symmetric about the center line of the weld, and the equation (4) is satisfied for the symmetric plane $y=0$, and the initial conditions of the energy conservation equation satisfy the formula (5).

$$
\frac{\partial T}{\partial y}=0 \quad T(x, y, z, 0)=T_{a}
$$

\subsection{Double Ellipsoid Heat Source Model}

The double ellipsoid body heat source put forward by Goldak which distribution mode is a kind of body heat source, that the spot welding arc heating source of heat power density distribution is moving double ellipsoid heat source model to describe[4]. The two parts of the body heat source on the work-piece are divided along the axis in order to better simulate the temperature gradient distribution of the front and back end of the moving heat source in the welding process. The specific mathematical expressions are as follows.

The internal heat flux density in the ellipsoid of the first half of the Y-axis is shown below.

$$
q_{\mathrm{f}}(x, y, z)=\frac{6 \sqrt{3}\left(f_{\mathrm{f}} \eta U I\right)}{a b_{1} c \pi \sqrt{\pi}} \exp \left(-\frac{3 x^{2}}{a^{2}}-\frac{3 y^{2}}{b_{1}^{2}}-\frac{3 z^{2}}{c^{2}}\right), \quad \mathrm{y} \geq 0
$$

The internal heat flux density of the ellipsoid in the second half of the Y-axis is shown below.

$$
q_{\mathrm{r}}(x, y, z)=\frac{6 \sqrt{3}\left(f_{\mathrm{r}} \eta U I\right)}{a b_{2} c \pi \sqrt{\pi}} \exp \left(-\frac{3 x^{2}}{a^{2}}-\frac{3 y^{2}}{b_{2}^{2}}-\frac{3 z^{2}}{c^{2}}\right), \quad \mathrm{y}<0
$$

Type: $\eta$ is thermal efficiency of arc. $I$ is the welding current; $U$ is the arc voltage; The distribution coefficient of the total input power in front of the molten pool and the latter two parts respectively; $a, b_{1}$, $\mathrm{b}_{2}$, and $\mathrm{c}$ are the double ellipsoid heat source shape parameters. See the literature [5].

\subsection{The Determination of the Heat Source Distribution Parameter}

First of all, according to the experience of the shape parameter initial value for the trial. Initial value can be selected according to the reach of the quasi steady state condition test value to the proportion of selected. For example, the measured a specific welding process conditions to macroscopic quasi steady state when molten pool length: molten pool width: the depth of molten pool $=8: 6: 1$, the double ellipsoid heat source the relationship between the shape parameters meet the $2 a:\left(b_{1}+b_{2}\right): c=6: 8: 1$, so can be preset $a, b_{1}, b_{2}, c=3: 3: 5: 1$, etc.

Will try to calculate the temperature field, comparing the results with the test results for specific welding process, the judge should adjust $a, b_{1}, b_{2}$, and $c$. Which of the shape parameter value is the parameter values increase or decrease; It is time to increase or decrease all shape parameter values $a, b_{1}$, $\mathrm{b}_{2}$, year on year, and finally determine the appropriate shape parameter value.

For heat source model is another important parameter -- $\mathrm{Q}_{0}$ input energy, the determination of $\mathrm{Q}_{0}$ also have certain rules to follow. Trial, an arbitrary input energy $\mathrm{Q}_{1}$, corresponding to get a final loaded into the work-piece on the output energy of $\mathrm{Q}_{2}$. According to the actual requirement for the output energy of $\mathrm{Q}=\eta \mathrm{UI}$, corresponding input energy $\mathrm{Q}_{0}$ and $\mathrm{Q}$ satisfies the following relationship.

$$
\frac{Q_{1}}{Q_{0}}=\frac{Q_{2}}{Q}
$$

Based on the calculation formula (8), the value of the input energy $\mathrm{Q}_{0}$ of the heat source is determined. 


\section{Finite Element Method}

Based on SYSWELD software, the above control equation and the definite solution conditions are solved by considering various factors such as evaporating heat dissipation of molten pool surface, convective heat dissipation in molten pool and the phase change latent heat of molten pool solid interface under the action of arc heat flow. The welding work-pieces for austenitic stainless steel $0 \mathrm{Cr} 18 \mathrm{Ni} 9$, size is $200 \mathrm{~mm} * 60 \mathrm{~mm} * 3 \mathrm{~mm}$. In the numerical simulation, the assumption that the work-piece surface and the heat transfer coefficient of air for $25 \mathrm{~W} /\left(\mathrm{m}^{2} \cdot \mathrm{K}\right)[5,6]$.

In order to reduce the calculation amount of the work-piece, due to the symmetry of the center line of the welded seam. To ensure accurate temperature distribution in the weld and its vicinity, the weld area is divided into the dense mesh, and the other areas are the grid, as shown in figure 1a. In order to better describe the distribution of the $3 \mathrm{~d}$ welding temperature field, the work-piece is divided into 25,900 units, and 23,001 nodes as shown in figure 1.

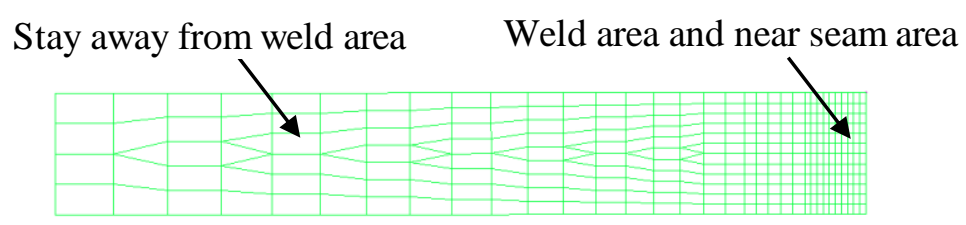

Fig. 1 Finite element grid schematic diagram

\section{Calculation Results and Analysis}

Using the corrected double ellipsoid thermal source model, the finite element numerical simulation of the concrete GTAW welding technology is carried out by using the corrected double ellipsoid thermal source model. Using I=110A, $U=12 \mathrm{~V}, \mathrm{v}=2 \mathrm{~mm} / \mathrm{s}$ welding conditions, the welding thermal efficiency $\eta=$ 0.7 numerical calculation. Figure $2 \mathrm{a}$ molten pool shape on the work-piece surface dimension transient changes over time. Figure $2 \mathrm{~b}$ describes the dynamic evolution process under the surface of molten pool shape work-piece. It can be seen that in 5.40 seconds the surface melts and the work-piece melts, which can be seen from both figure $2 \mathrm{c}$ and $2 \mathrm{~d}$. Figure $2 \mathrm{c}$ and $2 \mathrm{~d}$ respectively weld cross section, longitudinal and cross section shape of molten pool size change over time, you can see, with the extension of time, the molten pool grew older, weld width, length and depth of molten pool are gradually increasing, 7.47 seconds when molten pool shape to macroscopic quasi steady state.

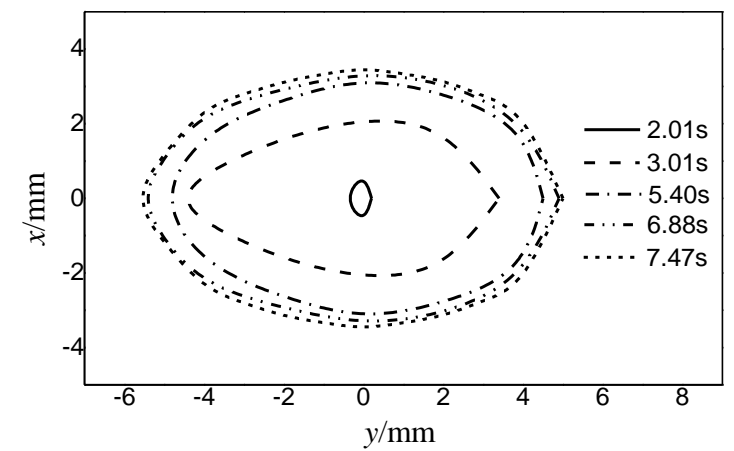

(a) Top surface

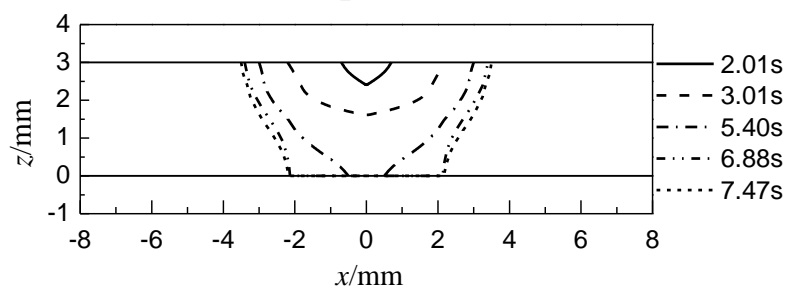

(c) Cross section

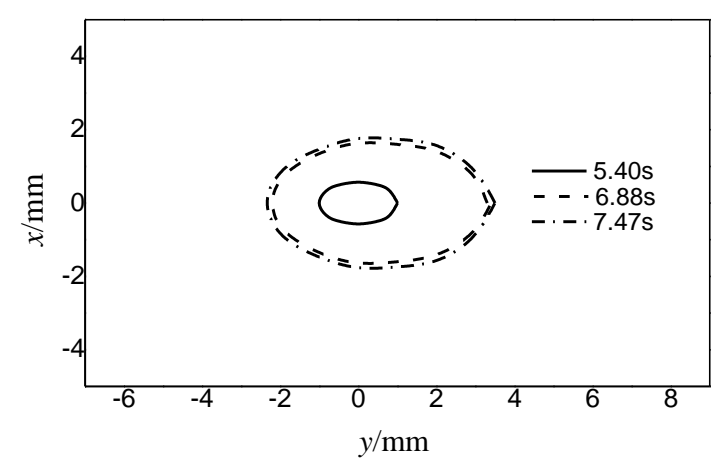

(b) Bottom surface

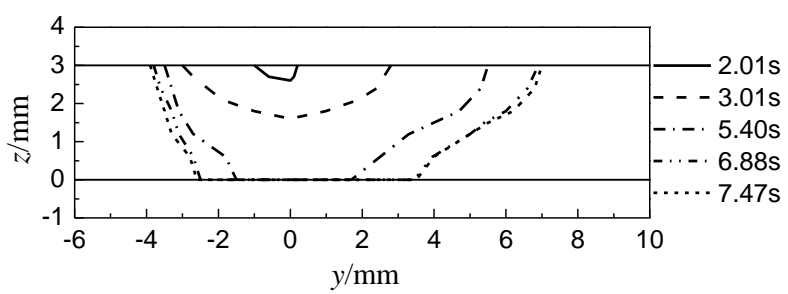

(d) Longitudinal section

Fig. 2 The dynamic evolution of the molten pool shape 
The heat from the molten pool depends on the size and temperature of the molten pool and the temperature around the molten pool. The higher the temperature, the faster the heat sinks. The higher the temperature of the molten pool is, the slower the heat dissipation is. Welding heat input is fixed value. At the beginning of welding, the molten pool is smaller and the temperature in the molten pool is lower, so the heat loss of the molten pool is less. The suction heat input is fixed. At the beginning of welding, the molten pool is smaller and the temperature in the molten pool is lower, so the heat loss of the molten pool is less. The heat of absorption is greater than that of the lost heat. The molten pool gradually grows up and the heat loss is gradually increased. Until the lost heat is in balance with the heat absorbed, the shape of the molten pool reaches the quasi-steady state.

Figure 3 is a comparison of the calculated value of the weld cross section of the welded stainless steel test piece in the case of a kind of GTAW welding stainless steel. It can be seen that in the case of free surface deformation of the molten pool, the shape and direction of the upper and lower surface melt and the fusion line are in the work-piece, and the calculated results are basically in line with the experimental results.

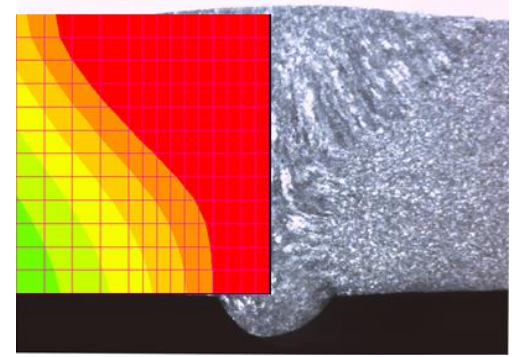

Fig. 3 Comparison between the predicted value and the test value

\section{Conclusion}

(1) Based on SYSWELD software, the finite element numerical analysis model of the welding temperature field and the stress field of the GTAW welding sheet of stainless steel sheet was established under the action arc. In this paper, the method to determine the distribution parameters of double ellipsoid body heat source is presented by using the calibration tool of SYSWELD software.

(2) Using the model of GTAW molten pool shape starting from the molten pool formed to reach macroscopic quasi steady state method to forecast the dynamic evolution process, the results show that reach macroscopic quasi steady state, the calculation result was consistent with the test result.

\section{References}

[1]. Ushio M, Wu C S. Mathematical modeling of 3-D heat and fluid flow in a moving GMA weld pool [J]. Metallurgical and Materials Transactions B, 1997, 28(2): 509-516.

[2]. Hirata Y, Asai Y, Takenak K, et al. 3-D numerical model predicting penetration shape in GTA welding [J]. Materials Science Forum, 2003, 11(5), 426-432.

[3]. Zhao P C, Wu C S, Zhang Y M. Numerical simulation of dynamic characteristic of weld pool geometry with step changes of welding parameters [J]. Modeling and Simulation in Materials Science and Engineering, 2004, 12(7): 765-780.

[4]. Goldak J, Chakravarti A, Bibbv M. A new finite element model for welding heat sources [J]. Metallurgical Transactions B, June, 1984, vol. 15B, 299-305.

[5]. Li R Y, Zhao M, Sun Y X. Finite element analysis of 3-D temperature fields on stainless steel sheet TIG welding based on SYSWELD software [J]. Hot working technology, 2007, 36(19): 69-72.

[6]. Li R Y, Zhao M, Zhou H Y. Finite element analysis on 3-D molten pool geometry for GTAW based on SYSWELD software [J]. Transaction of the China Welding Institution, 2011, 32(4): 4144. 